\title{
Antipsychotic medication induced movement disorders: The case of Amanuel Specialized Mental Hospital, Addis Ababa, Ethiopia
}

\author{
Habtamu Taye ${ }^{1, *}$, Tadesse Awoke², Jemal Ebrahim ${ }^{3}$ \\ ${ }^{1}$ Benishangul Gumuz Regional Health Bureau, Assosa, Ethiopia \\ ${ }^{2}$ Department of Epidemiology and Biostatistics, Institute of Public Health, College of Medicine and Health Science, University of Gondar, \\ Gondar, Ethiopia \\ ${ }^{3}$ Department of Psychiatry, College of Medicine and Health Sciences, Hawassa University, Hawassa, Ethiopia
}

Email address:

hab.taye@yahoo.com (H. Taye), tawoke7@gmail.com (T. Awoke), jemebrie@gmail.com (J. Ebrahim)

\section{To cite this article:}

Habtamu Taye, Tadesse Awoke, Jemal Ebrahim. Antipsychotic Medication Induced Movement Disorders: The Case of Amanuel Specialized Mental Hospital, Addis Ababa, Ethiopia. American Journal of Psychiatry and Neuroscience. Vol. 2, No. 5, 2014, pp. 76-82.

doi: 10.11648/j.ajpn.20140205.12

\begin{abstract}
Background: Neuroleptic-induced movement disorders constitute a worldwide problem in the treatment of schizophrenia because of the limited affordability of atypical antipsychotic drugs. The observable features of acute Parkinsonism; such as limb stiffness and slowness of movement are a social and functional handicap. The same is true for the restless movements and agitation associated with acute akathisia. Tardive dyskinesia, on the other hand is a permanent condition that affects quality of life. However, very few studies have been conducted to estimate the prevalence of Neurolepticinduced movement disorders and their associated factors among psychotic patients in Ethiopia. Thus the aim of this study was to determine the prevalence of conventional antipsychotic induced movement disorders and associated factors among psychotic patients treated at Amanuel mental specialized Hospital. Method: Hospital based cross-sectional study was conducted by using established clinical rating scales to identify cases of conventional antipsychotic-induced movement disorders in Amanuel mental specialized Hospital on a sample of 377 psychotic outpatients. Systematic random sampling method was employed to select subjects. Logistic regression was used for comparison of the subjects with and without Neuroleptic-induced movement disorders. Results: the prevalence of Neuroleptic-induced movement disorders, namely; neuroleptic-induced Parkinsonism, neuroleptic-induced Akathisia and neuroleptic-induced tardive dyskinesia were found to be 46.4\%, 28.6\% and 11.9\% respectively. Khat (Catha Edulis) use, AOR=1.93, 95\%CI: 1.01-3.66, was factors remained to be associated with the presence of NIA. Alcohol use, AOR $=3.25,95 \% \mathrm{CI}$ : 1.04-10.16, was associated with TD. Being on chlorpromazine equivalent dose range of $>=400 \mathrm{mg} / \mathrm{day}$, $\mathrm{AOR}=4.32,95 \% \mathrm{CI}$ : $2.25-8.30$, $\mathrm{AOR}=3.677,95 \% \mathrm{CI}$ : $1.807-7.482$, $\mathrm{AOR}=4.157$, 95\%CI: 1.165-14.834 were associated with Parkinsonism, Akathisia and TD respectively. Conclusions and Recommendation: Considerable number of patients with psychotic disorders suffered from a conventional antipsychotic induced movement disorder. Khat, alcohol and high dose of drugs were found to be associated with conventional antipsychotic Induced movement disorders. Designing treatment guideline, increasing availability of drugs with minimal side effects and psycho-education for patients and their family is essential to reduce these devastating side effects.
\end{abstract}

Keywords: Parkinsonism, Akathisia, Tardive Dyskinesia, Conventional Antipsychotic, Movement Disorder

\section{Introduction}

Antipsychotic drugs include dopamine receptor antagonists or conventional antipsychotics, serotonin-dopamine antagonists or atypical antipsychotics and dopamine partial agonists [1].
Since their development in 1950s conventional antipsychotic medications have been a primary component of treatment for schizophrenia and related psychotic disorders [2]. The therapeutic efficacy of these drugs is well established, both for treatment of acute symptoms and in relapse prevention. Unfortunately, they are associated with a broad range of side-effects, the most prominent of which is 
the development of a variety of movement disorders [3]. Factors for Tardive dyskinesia were Old age, Female sex, Brain damaged individuals, cumulative neuroleptic dose, duration of exposure, Presence of drug induced Parkinsonism in the early phase of neuroleptic treatment, Primary psychiatric diagnosis of affective disorder and substances including alcohol $[3,4,5]$. The risk factors leading to akathisia were poorly understood; though it is noted more with high potency antipsychotics possibly due to employment of higher doses. Middle aged Women are at greatest risk [6, 7 , 8].

Risk Factors for Development of Drug-Induced Parkinsonism were High dose, high-potency drug use; elderly, female sex, hereditary susceptibility and Coexistence with tardive dyskinesia $[4,8]$.

Cross-sectional Study on institutionalized psychotic patients from Central Estonia reported that, 31.3\% had neuroleptic-induced akathisia $23.2 \%$ had neuroleptic-induced Parkinsonism and 32.3\% had neuroleptic-induced tardive dyskinesia [9]. Previous reports regarding the association of Akathisia with socio-demography were inconsistent. Some of them reported that there was no significant difference in age between the Akathisia and non-Akathisia groups [10,11, and 12]. The relationship between Akathisia and sex has also been inadequately investigated [14]. Some studies reported higher prevalence is among females [6, 13 and 15]. Most epidemiological studies have not reported any sex differences in the vulnerability to Akathisia [11, 12, and 16]. Another study on the other hand, stated that "Akathisia...tends to prevail in men"[17]. Review article from UK concluded that there was no significant gender difference for development of drug induced Parkinsonism but there are reports that stated the male to female ratio is $1: 2[4,18]$. Nigerian Study done in 1981 reported the prevalence rate of TD was higher among the females $(14.5 \%)$ than among the males [7\%]. All TD cases were found to be of an older age group than the total population [19]. However study conducted in 1989 reported there were no significant differences between the sexes [20].

Search for literature revealed, there was no study that investigated the prevalence of conventional antipsychotic induced movement disorders and associated factors in Ethiopia.

Despite mental health problem was included in national health policy of Ethiopia, interventions against the problem are limited. Among the main reasons, lack of data on the extent of the problem is one. Therefore this study was aimed to determine the prevalence of conventional antipsychotic induced movement disorders and associated factors among Psychotic patients attending treatment at Amanuel Hospital, Addis Abeba, Ethiopia. It was also hoped that this study would increase the awareness of clinicians to critically look and treat patients with antipsychotic induced movement disorders; help administrators to investigate solutions for the problems and serve as base line for those who wish to conduct study on antipsychotic induced movement disorders

\section{Methodology}

Hospital based cross-sectional study was conducted from February 9 to March 9/2011 among psychotic patients treated at Amanuel mental specialized Hospital. Systematic random Sampling technique was employed. Five experienced psychiatric nurses were hired and assessed all subjects after being trained to identify the movement disorders in accordance with Barnes Rating Scale for Akathisia (8), the Simpson-Angus Rating Scale for Drug-Induced Parkinsonism [9] and Abnormal Involuntary Movement Scale (AIMS) for Tardive dyskinesia [10]. The threshold value for Akathisia was a Barnes scale total score of 2 or more (scale range $=0-5$ ); for Parkinsonism, the threshold value was a Simpson-Angus Rating Scale mean global score of 0.65 or more (scale range $=0-4)$. For the diagnosis of Tardive dyskinesia, a minimum global rating of "mild" (i.e. 2 or more on AIMS item no. 8), was used.

For analysis, Coded variables were entered into SPSS version 13.0 window software program. Logistic regression was used for comparison of subjects with and without conventional antipsychotic-induced movement disorders. From the bivariate analysis three different models were fitted for the three outcome variables in relation to each explanatory variable. Those which fulfilled the minimum requirement, 0.2 level of significance; were entered in to multivariate logistic analysis for further assessment. Fitness of model check showed that, the model adequately fitted the data for Neuroleptic Induced Parkinsonism, Neuroleptic Induced Akathisia, and Tardive dyskinesia as P- value from Hosmer and Lemeshow test was $0.86,0.78$ and 0.82 respectively. Written informed consent was obtained from the subjects, and the study was approved by University of Gondar College of medicine and health science ethical committee. The main limitations of this study were: Co-occurrence of spontaneous movement disorders commonly detected in schizophrenic population which could not be excluded.

\section{Results}

Of the 403 planned participants, 93.5\% were involved in the study of which, 65.3 were males. Twenty six patients failed to fulfill the inclusion criteria. The median age of participants was 30.0 years $(\mathrm{SD}=10.57)$. Of the total participants the majority; 126(33.4\%) were Amhara, 274(72.7\%) were Christians, 93(24.7) were jobless, $184(48.8 \%)$ were within primary school educational levels and $234(62.1 \%$ ) were single in marital status. (Table 1)

Of the 377 participants $80.6 \%$ had the diagnosis of schizophrenia, $58.4 \%$ had gone $<5$ years with their illness and $9.0 \%$ had family history of primary movement disorders. High potent antipsychotic drugs were taken by $41.4 \%$ of participants. Patients on Chlorpromazine equivalent dose range of $>=400 \mathrm{mg} /$ day were $32 \%$ and $15.38 \%$ participants took combination of antipsychotic drugs. Of these $13.3 \%$ of them took Fluphenazine and chlorpromazine combination treatment. (Table 2) 
Table 1. Distribution of participants by socio-demographic Characteristics at Amanuel mental specialized Hospital, 2011.

\begin{tabular}{lll}
\hline Characteristics & Frequency (N=377) & Percent (\%) \\
\hline Age & 172 & \\
$<30$ & 142 & 45.6 \\
$30-44$ & 63 & 37.67 \\
$>=45$ & & 16.71 \\
Sex & 246 & \\
Male & 131 & 65.3 \\
Female & & 34.7 \\
Educational status & 68 & \\
Can't read and write & 125 & 18.0 \\
1-6 grades & 71 & 33.2 \\
$7-12$ grades & 113 & 18.8 \\
$>12$ grades & & 30.0 \\
Religion & 274 & \\
Christian & 103 & 72.7 \\
Muslim & & 27.3 \\
Marital Status & 103 & \\
Married & 234 & 27.3 \\
Single & 40 & 62.1 \\
Divorced/widowed & & 10.6 \\
Ethnicity & 96 & \\
Oromo & 126 & 33.4 \\
Amhara & 107 & 28.4 \\
Gurage & 48 & 12.7 \\
Others & & 75.3 \\
Occupation & 284 & \\
With job & & \\
\hline
\end{tabular}

Table 2. Distribution of participants by clinical Characteristics at Amanuel mental specialized Hospital, 2011.

\begin{tabular}{lll}
\hline Characteristics & $\begin{array}{l}\text { Frequency } \\
(\mathbf{N}=\mathbf{3 7 7})\end{array}$ & Percent (\%) \\
\hline Diagnosis of patients & & \\
Schizophrenia & 304 & 80.6 \\
Schizoaffective disorder & 22 & 5.8 \\
Schizophreniform disorder & 23 & 6.1 \\
Others & 28 & 7.4 \\
Family history of primary & & \\
movement disorders & & \\
Yes & 34 & 9.0 \\
No & 343 & 91.0 \\
Chlorpromazine equivalent dose & & \\
(mg/day) & & \\
50-<100 & 118 & 31.3 \\
100-<400 & 138 & 36.6 \\
$>=400$ & 121 & 32.1 \\
High potent antipsychotic drugs & & \\
Yes & 156 & 41.4 \\
No & 221 & 58.6 \\
\hline
\end{tabular}

Among participants using substances; 7.7\% consumed alcohol, 24.4\% smoked cigarette and $28.1 \%$ chewed khat.

\subsection{Prevalence of Conventional Antipsychotic Induced Movement Disorders}

The overall prevalence of conventional antipsychotic induced movement disorders was 56\%: neuroleptic induced Parkinsonism 46.4, Akathisia $28.6 \%$ and tardive dyskinesia $11.9 \%$ respectively. (Fig. 1)

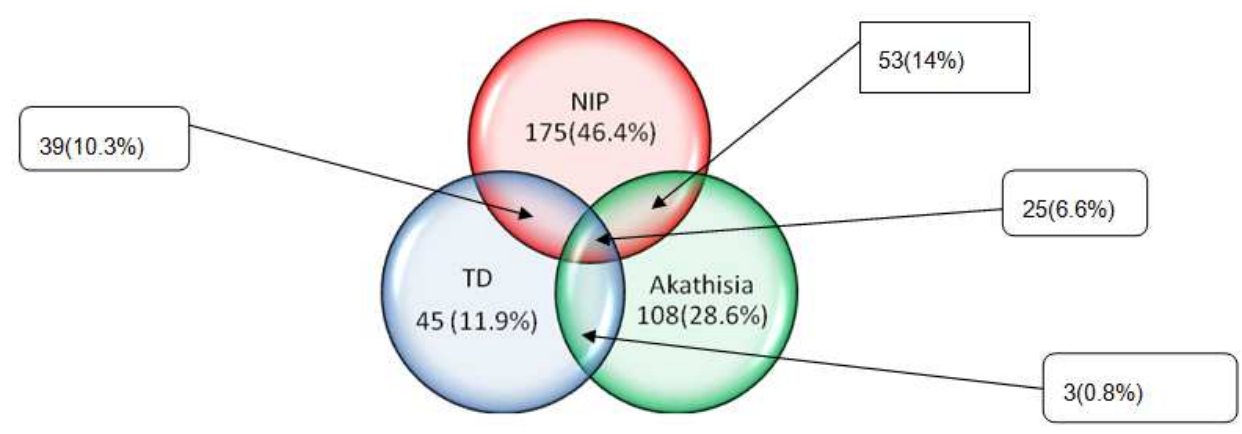

Fig 1. Prevalence of Neuroleptic induced movement disorders among psychotic patients treated at Amanuel mental specialized Hospital, 2011.

\subsection{Factors Associated with Antipsychotic Induced Movement Disorders}

During analysis of neuroleptic induced parkinsonism in relation to all explanatory variables, being on chlorpromazine equivalent dose range of $100-<400 \mathrm{mg} /$ day $\mathrm{AOR}=2.18$, 95\%CI: $1.22-3.88$ and $>=400 \mathrm{mg} /$ day $\mathrm{AOR}=4.32,95 \% \mathrm{CI}$ : 2.25-8.30 as well as being on high potent conventional antipsychotics $\mathrm{AOR}=4.63,95 \% \mathrm{CI}): 1.93-11.13$ were factors remained to be statistically significant. (Table 3 )

During the multivariate analysis of Akathisia in relation to all exploratory variables, khat use $\mathrm{AOR}=1.93,95 \% \mathrm{CI}$ : 1.01 -
3.66 and being on chlorpromazine equivalent dose range of $100-<400 \mathrm{mg} /$ day $\quad \mathrm{AOR}=2.27, \quad 95 \% \mathrm{CI}$ : $1.16-4.43$ and $>=400 \mathrm{mg} /$ day $\mathrm{AOR}=3.68,95 \% \mathrm{CI}: 1.81-7.48$ were factors remained to be statistically significant. (Table 4)

During the analysis of TD; age $>=45$ years $\mathrm{AOR}=3.45$, 95\%CI: $1.12-10.63$, joblessness $\mathrm{AOR}=3.19$, 95\%CI: 1.43 7.16, alcohol use AOR $=3.25$, 95\%CI: 1.04-10.16, and chlorpromazine equivalent dose range of $>=400 \mathrm{mg} /$ day $\mathrm{AOR}=4.157,95 \% \mathrm{CI}: 1.17-14.83$ were the most contributing factors remained to be statistically significant. (Table 5) 
Table 3. Factors associated with neuroleptic induced Parkinsonism (Bivariate analyses and multivariate analysis), at Amanuel mental specialized Hospital, among psychotic patients, 2011.

\begin{tabular}{|c|c|c|c|c|}
\hline \multirow{2}{*}{ Explanatory variable } & \multicolumn{2}{|c|}{ Parkinsonism ( $\mathrm{N}=\mathbf{3 7 7}$ ) } & \multirow{2}{*}{ COR (95\%C.I.) } & \multirow{2}{*}{ AOR (95\%C.I.) } \\
\hline & Yes & No & & \\
\hline \multicolumn{5}{|l|}{ Ethnicity } \\
\hline Oromo & 41 & 55 & 1.00 & 1.00 \\
\hline Amhara & 50 & 76 & $0.88(0.52,1.51)$ & $0.71(0.38,1.30)$ \\
\hline Guragie & 62 & 45 & $1.85(1.06,3.23)^{*}$ & $1.42(0.74,2.74)$ \\
\hline Others & 22 & 26 & $1.14(0.57,2.28)$ & $0.88(0.39,1.99)$ \\
\hline \multicolumn{5}{|l|}{ Marital status } \\
\hline Married & 36 & 67 & 1.00 & 1.00 \\
\hline Single & 118 & 116 & $1.89(1.17,3.06)^{*}$ & $1.57(0.90,2.72)$ \\
\hline Divorced/widowed & 21 & 19 & $2.06(0.98,4.32)$ & $1.54(0.65,3.65)$ \\
\hline \multicolumn{5}{|l|}{ Duration of treatment } \\
\hline 1month- $<6$ month & 94 & 138 & 1.00 & 1.00 \\
\hline 6month-5yr & 54 & 42 & $1.89(1.17,3.05)^{*}$ & $1.24(0.70,2.18)$ \\
\hline$>=5 \mathrm{yrs}$ & 27 & 22 & $1.80(0.97,3.35)$ & $1.28(0.58,2.84)$ \\
\hline \multicolumn{5}{|l|}{ Alcohol use } \\
\hline Yes & 18 & 11 & $1.99(0.91,4.34)$ & $1.45(0.54,3.88)$ \\
\hline No & 157 & 191 & 1.00 & 1.00 \\
\hline \multicolumn{5}{|l|}{ Cigarette use } \\
\hline Yes & 57 & 35 & $2.31(1.42,3.73)^{*}$ & $1.29(0.65,2.53)$ \\
\hline No & 118 & 167 & 1.00 & 1.00 \\
\hline \multicolumn{5}{|l|}{ Khat use } \\
\hline Yes & 65 & 41 & $2.32(1.47,3.68)^{*}$ & $1.45(0.76,2.75)$ \\
\hline No & 110 & 161 & 1.00 & 1.00 \\
\hline \multicolumn{5}{|c|}{ High potent antipsychotic drugs } \\
\hline Yes & 100 & 56 & $3.48(2.26,5.34)^{*}$ & $2.30(1.400,3.77) *$ \\
\hline No & 75 & 146 & 1.00 & 1.00 \\
\hline \multicolumn{5}{|c|}{ Chlorpromazine equivalent dose(mg/day) } \\
\hline $50-<100$ & 28 & 90 & 1.00 & 1.00 \\
\hline $100-<400$ & 61 & 77 & $2.55(1.48,4.37)^{*}$ & $2.34(1.31,4.15)^{*}$ \\
\hline$>=400$ & 86 & 35 & $7.90(4.43,14.08)^{*}$ & $5.13(2.72,9.68)^{*}$ \\
\hline
\end{tabular}

* Statistically significant

Table 4. Factors associated with neuroleptic induced Akathisia (Bivariate analyses and multivariate analysis), at Amanuel mental specialized Hospital, among psychotic patients, 2011.

\begin{tabular}{|c|c|c|c|c|}
\hline \multirow{2}{*}{ Explanatory variable } & \multicolumn{2}{|c|}{ Akathisia(N=377) } & \multirow{2}{*}{ COR (95\%C.I.) } & \multirow{2}{*}{ AOR (95\%C.I.) } \\
\hline & Yes & No & & \\
\hline \multicolumn{5}{|l|}{ Age } \\
\hline$<30$ & 43 & 129 & 1.00 & 1.00 \\
\hline $30-44$ & 42 & 100 & $1.26(0.77,2.08)$ & $1.21(0.69,2.11)$ \\
\hline$>=45$ & 23 & 40 & $1.73(0.93,3.20)$ & $1.44(0.72,2.86)$ \\
\hline \multicolumn{5}{|l|}{ Sex } \\
\hline Male & 78 & 168 & 1.00 & 1.00 \\
\hline Female & 30 & 101 & $0.64(0.39,1.04)$ & $1.01(0.57,1.78)$ \\
\hline \multicolumn{5}{|l|}{ Occupation } \\
\hline With job & 74 & 210 & 1.00 & 1.00 \\
\hline Jobless & 34 & 59 & $1.64(0.99,2.69)$ & $1.63(0.94,2.85)$ \\
\hline \multicolumn{5}{|l|}{ Diagnosis of patients } \\
\hline Schizophrenia & 92 & 212 & 1.00 & 1.00 \\
\hline Others & 16 & 57 & $0.65(0.35,1.19)$ & $0.66(0.33,1.31)$ \\
\hline \multicolumn{5}{|l|}{ Alcohol use } \\
\hline Yes & 14 & 15 & $2.52(1.17,5.42)^{*}$ & $1.68(0.68,4.14)$ \\
\hline No & 94 & 254 & 1.00 & 1.00 \\
\hline \multicolumn{5}{|l|}{ Cigarette use } \\
\hline Yes & 37 & 55 & $2.03(1.24,3.33)^{*}$ & $0.93(0.47,1.85)$ \\
\hline No & 71 & 214 & 1.00 & 1.00 \\
\hline \multicolumn{5}{|l|}{ Khat use history } \\
\hline Yes & 45 & 61 & $2.44(1.51,3.93)^{*}$ & $1.93(1.01,3.66)^{*}$ \\
\hline No & 63 & 208 & 1.00 & 1.00 \\
\hline \multicolumn{5}{|l|}{ Cannabis use history } \\
\hline Yes & 5 & 1 & $13.01(1.50,112.70)^{*}$ & $5.93(0.63,55.63)$ \\
\hline No & 103 & 268 & 1.00 & 1.00 \\
\hline \multicolumn{5}{|c|}{ High potent antipsychotic drugs } \\
\hline Yes & 54 & 102 & $1.64(1.04,2.57)^{*}$ & $1.05(0.62,1.80)$ \\
\hline
\end{tabular}




\begin{tabular}{lllll}
\hline \multirow{2}{*}{ Explanatory variable } & \multicolumn{2}{l}{ Akathisia(N=377) } & \multirow{2}{*}{ COR (95\%C.I.) } & \multirow{2}{*}{ AOR (95\%C.I.) } \\
\cline { 2 - 3 } & Yes & No & 1.00 & 1.00 \\
No & 54 & 167 & & 1.00 \\
Chlorpromazine equivalent dose(mg/day) & 16 & 102 & 1.00 & $2.27(1.16,4.43)^{*}$ \\
$50-<100$ & 41 & 97 & $2.70(1.42,5.12)^{*}$ & $3.68(1.81,7.48)^{*}$ \\
$100-<400$ & 51 & 70 & $4.65(2.452,8.80)^{*}$ & \\
$>=400$ & &
\end{tabular}

*Statistically significant

Table 5. Factors associated with neuroleptic induced Tardive dyskinesia (Bivariate analyses and multivariate analysis), at Amanuel mental specialized Hospital, among psychotic patients, 2011.

\begin{tabular}{|c|c|c|c|c|}
\hline \multirow{2}{*}{ Explanatory variable } & \multicolumn{2}{|c|}{ Tardive dyskinesia $(\mathrm{N}=377)$} & \multirow{2}{*}{ COR(95\%C.I.) } & \multirow{2}{*}{ AOR(95\%C.I.) } \\
\hline & Yes & No & & \\
\hline \multicolumn{5}{|l|}{ Age } \\
\hline$<30$ & 9 & 163 & 1.00 & 1.00 \\
\hline $30-44$ & 15 & 127 & $2.14(0.91,5.05)$ & $1.19(0.45,3.16)$ \\
\hline$>=45$ & 21 & 42 & $9.06(3.87,21.22)^{*}$ & $3.45(1.12,10.63)^{*}$ \\
\hline \multicolumn{5}{|l|}{ Occupation } \\
\hline With job & 27 & 257 & 1.00 & 1.00 \\
\hline Jobless & 18 & 75 & $2.28(1.19,4.37)^{*}$ & $3.19(1.43,7.16)^{*}$ \\
\hline \multicolumn{5}{|l|}{ Marital status } \\
\hline Married & 15 & 88 & 1.00 & 1.00 \\
\hline Single & 21 & 213 & $0.58(0.29,1.17)$ & $0.65(0.25,1.71)$ \\
\hline Divorced/widowed & 9 & 31 & $1.70(0.68,4.28)$ & $0.85(0.28,2.60)$ \\
\hline \multicolumn{5}{|c|}{ Duration of the psychotic disorder } \\
\hline$<5$ yrs & 10 & 211 & 1.00 & 1.00 \\
\hline $5-9 \mathrm{yrs}$ & 13 & 58 & $4.73(1.97,11.34)^{*}$ & $4.92(0.98,24.72)$ \\
\hline $10-19$ & 18 & 51 & $7.45(3.24,17.10)^{*}$ & $3.09(0.44,21.87)$ \\
\hline$>=19$ & 4 & 12 & $7.03(1.92,25.74)^{*}$ & $2.45(0.20,29.74)$ \\
\hline \multicolumn{5}{|l|}{ Duration of treatment } \\
\hline 1 month-<6month & 13 & 219 & 1.00 & 1.00 \\
\hline 6month-5yr & 15 & 81 & $3.12(1.42,6.84)^{*}$ & $0.64(0.13,3.20)$ \\
\hline$>=5 \mathrm{yrs}$ & 17 & 32 & $8.95(3.97,20.15)^{*}$ & $2.06(0.30,14.35)$ \\
\hline \multicolumn{5}{|l|}{ Alcohol use } \\
\hline Yes & 10 & 19 & $4.71(2.03,10.92)^{*}$ & $3.25(1.04,10.16)^{*}$ \\
\hline No & 35 & 313 & 1.00 & 1.00 \\
\hline \multicolumn{5}{|l|}{ Cigarette use } \\
\hline Yes & 19 & 73 & $2.59(1.36,4.95)^{*}$ & $1.54(0.66,3.55)$ \\
\hline No & 26 & 259 & 1.00 & 1.00 \\
\hline \multicolumn{5}{|c|}{ High potent antipsychotic drugs } \\
\hline Yes & 24 & 132 & $1.73(0.93,3.24)$ & $0.85(0.35,2.06)$ \\
\hline No & 21 & 200 & 1.00 & 1.00 \\
\hline \multicolumn{5}{|c|}{ Chlorpromazine equivalent dose(mg/day) } \\
\hline $50-<100$ & 5 & 113 & 1.00 & 1.00 \\
\hline $100-<400$ & 16 & 122 & $2.96(1.05,8.35)^{*}$ & $2.50(0.77,8.11)$ \\
\hline$>=400$ & 24 & 97 & $5.59(2.06,15.22)^{*}$ & $4.16(1.17,14.83)^{*}$ \\
\hline
\end{tabular}

* Statistically significant

\section{Discussion}

Hospital based cross sectional study was conducted to assess magnitude of conventional antipsychotic induced movement disorders and associated factors by using clinical rating scales (SAS, BAS and AIMS). The overall prevalence of 56\%: neuroleptic induced Parkinsonism 175(46.4\%), Akathisia 108(28.6\%) and tardive dyskinesia $45(11.9 \%)$ were found respectively. The results were slightly different from previous studies done abroad [20, 21 and 22]. Some of the variations were probably due to variability of the disorders themselves. Individual patients may show a wide variability in site and severity of involuntary movements, related to adjustment of medication, anxiety, posture and mobility [2].

Even though, giving combination of the same class of drugs was not medically explained; $15.38 \%$ of participants took combination of conventional antipsychotic drugs. Of these $13.3 \%$ of them took Fluphenazine and chlorpromazine combination treatment.

During the multivariate analysis of NIP in relation to all explanatory variables, being on chlorpromazine equivalent dose range of $100-<400 \mathrm{mg} /$ day and $>=400 \mathrm{mg} /$ day had 2.18 and 4.32 times more likelihood of inducing NIP than lesser doses. Being on high potent conventional antipsychotics had 4.63 times chance of inducing NIP. This result was consistent with previous studies [17, 22].

Absence of association between gender and Parkinsonism was consistent with other studies [11, 18]. Similar to Estonian study there was no differences between patients with a neuroleptic-induced movement disorder and those 
without with respect to sex, smoking status \& length of illness and treatment [2].

During the multivariate analysis of NIA in relation to all explanatory variables, khat users had 1.93 times likelihood of having NIA compared to non users.

Possible explanations could be:

1. Akathisia was assumed to be caused by blockage of dopamine D2 receptor in nigrostriatal pathway and khat which is assumed to have similar action with Amphetamine increases striatal dopamine release in acute phase. However, its chronic effects are associated with striatal dopaminergic downregulation which can potentiate antipsychotic action.

2. Patients who took khat may develop symptoms similar with Akathisia on withdrawal phase.

Being on chlorpromazine equivalent dose of 100$<400 \mathrm{mg} /$ day and $>=400 \mathrm{mg} /$ day had 2.27 and 3.68 times chance of inducing Akathisia. This was in line with studies done elsewhere $[17,22]$. Absence of statistical difference between patients with Akathisia and those without, with respect to sex, age, ethnicity, education, religion, marital status, occupational status, alcohol use, cigarette use, diagnosis of patient's duration of illness and duration of treatment. This was consistent with previous studies with respect to sex $[25,26,30]$, age $[25,26]$, ethnicity [23] and smoking status [2].

During the analysis of TD in relation to all explanatory variables; age $>=45$ were 3.45 times more likely to develop TD. This was consistent with multiple studies [19, 22, and 27]. Alcohol users had 3.25 times more likelihood of developing TD. This was consistent with some studies [24, 25] and being on chlorpromazine equivalent dose range $>=400 \mathrm{mg} /$ day had 4.16 times more likelihood to induce TD. This was similar with other studies [24]. Lack of evidence for diagnostic specificity as a predictor for the occurrence of TD was consistent with some studies [23].

This study was not without limitations. Reports for some of the questions were past history or encounters which are prone to recall bias. Variables like Khat chewing and other substances are by nature a sensitive issue and social desirability bias is unavoidable. Co-occurrence of spontaneous movement disorders, commonly detected in schizophrenic populations, could not be excluded. The temporal connection between NIMD and neuroleptic medication as well as other association was established retrospectively by interview and medical records.

\section{Conclusions and Recommendation}

In conclusion, many patients with psychotic disorders suffered from a conventional antipsychotic -induced movement disorders which were seen as burdening and stigmatizing phenomena.

All of the antipsychotic induced movement disorders were found to be associated with chlorpromazine equivalent doses. Khat which was thought to be indigenous to Ethiopia is consumed by many patients who had been on antipsychotic drugs and found to be associated with Akathisia; one of distressing side effect.

Designing treatment guideline, increasing availability of drugs with minimal side effects and psycho-education on associated factors (e.g. khat use, alcohol consumption) is essential.

\section{Authors' contribution}

HT and TA designed the study, collected data and participated on data analysis and preparation of the manuscript. JE participated in analysis, drafted and prepared the manuscript. All the authors approved the final manuscript.

\section{Acknowledgement}

The authors would like to acknowledge Amanuel Specialized Mental Hospital for financial support. We also would like to thank the study subjects, data collectors and supervisors for their admirable endeavor during the data collection process.

\section{References}

[1] Lieberman JA. Dopamine partial agonists: a new class of antipsychotic. CNS Drugs. 2004; 18(4):251-67.

[2] Marion A. Becker, M. Scott Young, Ezra Ochshorn and Ronald J. Diamond. The Relationship of Antipsychotic Medication Class and Adherence with Treatment Outcomes and Costs for Florida Medicaid Beneficiaries with Schizophrenia. Administration and Policy in Mental Health and Mental Health Services Researchm. 2007; 34(3):307314.

[3] Maurice Gervin and Thomas R.E. Barnes. Assessment of drug-related movement disorders in schizophrenia. Advances in Psychiatric Treatment. 2000; 6(5): 332-341.

[4] John Muench; Ann M Hamer. Adverse effects of antipsychotic medications. American family physician.2010; 81(5):585-589

[5] Sven Janno, M.D., Matti Holi, M.D., Ph.D., Katinka Tuisku, M.D., Ph.D., and Kristian Wahlbeck, M.D., Ph.D. Prevalence of Neuroleptic-Induced Movement Disorders in Chronic Schizophrenia Inpatients. The American journal of psychiatry. 2004; 161:160-163.

[6] Kapil D. Sethi, M.D., F.R.C.P.(UK). Movement Disorders Induced by Dopamine Blocking Agents. Seminars in Neurology. 2001; 21(1):61-65.

[7] A. Osman, M. Marghalani,I.Y.A. Turkistani,M. Al-Swaf and B. Bin Sadiq .Neuroleptic-induced tardive dyskinesia among Arab psychotic patients. WHO-EMRO- East Meditranian Health journal.2007; 13( 3).

[8] Barnes TRE: A rating scale for drug-induced akathisia. Br J Psychiatry. 1989; 154:672-676.

[9] Simpson GM, Angus JWS: A rating scale for extrapyramidal side effects. Acta Psychiatr Scand Suppl .1970; 212:11-19. 
[10] Guy W. Abnormal Involuntary Movement Scale (AIMS). ECDEU Assessment Manual for Psychopharmacology Revised. 1976; 76-338.

[11] American Psychiatric Association Diagnostic and Statistical Manual of Mental Disorders. Fourth. Washington D/C: American Psychiatric Press; 2000.

[12] Kane JM, Woerner M, Woerner M, Lieferman J. Tardive dyskinesia Prevalence, incidence and risk factors. J. C 'in Psychopharmacology. 1988; 8:52-56.

[13] Ramesh S, Sagar R. Antipsychotic induced movement disorders. Indian J Med Sci.2001;55(9):483-7.

[14] Michael P. Caligiuri, Dilip V. Jeste, and Jonathan P. Lacro. Antipsychotic-Induced Movement Disorders in the Elderly Epidemiology and Treatment Recommendations. Drugs \& Aging. 2000; 17(5):363-384.

[15] Braude, W.M.; Barnes, T.R.; and Gore, S.M. Clinical characteristics of akathisia: A systematic investigation of acute psychiatric inpatient admissions. British Journal of Psychiatry. $1983 ; 143: 139-150$.

[16] Sachdev $P$ and Kruk, J. Clinical characteristics and predisposing factors in acute drug-induced akathisia. Archives of General Psychiatry.1994; 51(12):963-974.

[17] Ayd, F., Jr. A survey of drug induced extrapyramidal reactions. Journal of the American Medical Association.1961; 175:10541060 .

[18] Permlnder Sachdev. The Epidemiology of Drug-Induced Akathisia: Part I. Acute Akathisia. Shizophrenia Bulletin. 1995; 21( 3) :441-443.

[19] Sarwer-Foner, G.J. Recognition and management of drug- induced extrapyramidal reactions and "paradoxical" behavioral reactions in psychiatry. Canadian Medical Association Journal.1960; 83:312-318.

[20] B Thanvi, S Treadwell. Drug induced Parkinsonism: a common cause of Parkinsonism in older people. Postgrad Med J. 2009; 85(1004):322-32

[21] Odejide AO. The prevalence of persistent abnormal involuntary movements among patients in a Nigerian long stay psychiatric unit. Afr J Med Med Sci. 1981;10(1-2):39-43.

[22] Gureje O. Tardive dyskinesia in schizophrenics. Prevalence, distribution and relationship to neurological "soft" signs in Nigerian patients. Acta Psychiatr Scand. 1987 ;76(5):523-8.

[23] Gureje O. The significance of subtyping tardive dyskinesia: a study of prevalence and associated factors. Psychol Med. 1989;19(1):121-8.

[24] Lane, R. D., Glazer, W. M., Hansen, T. E., et al Assessment of tardive dyskinesia using the abnormal involuntary movement scale. Journal of Nervous and Mental Disease. 1985; 173: 353-357.

[25] Sven Janno, Matti M Holi, Katinka Tuisku and Kristian Wahlbeck. Validity of Simpson-Angus Scale (SAS) in a naturalistic schizophrenia population.BMC Neurology. 2005; $5: 5$.

[26] S Potvin, et al. Increased extrapyramidal symptoms in patients with schizophrenia and a comorbid substance use disorder. J Neurol Neurosurg Psychiatry. 2006 ; 77(6): 796-798.

[27] Sven Janno. Assessment of neuroleptic-induced movement disorders in a naturalistic schizophrenia population. Helsinki University Printing House. 2006. 